\title{
Exact laws for sums of ratios of order statistics from the pareto distribution
}

\author{
André Adler* \\ Department of Mathematics, \\ Illinois Institute of Technology, \\ Chicago, Illinois, 60616, USA
}

Received 25 January 2005; accepted 29 September 2005

\begin{abstract}
Consider independent and identically distributed random variables $\left\{X_{n k}, 1 \leq k \leq\right.$ $m, n \geq 1\}$ from the Pareto distribution. We select two order statistics from each row, $X_{n(i)} \leq$ $X_{n(j)}$, for $1 \leq i<j \leq m$. Then we test to see whether or not Laws of Large Numbers with nonzero limits exist for weighted sums of the random variables $R_{i j}=X_{n(j)} / X_{n(i)}$.

(c) Central European Science Journals Warsaw and Springer-Verlag Berlin Heidelberg. All rights reserved.
\end{abstract}

Keywords: Almost sure convergence, weak law of large numbers, strong law of large numbers, order statistics, exact laws

MSC (2000): 60F05, 60F15

In this paper we observe weighted sums of ratios of order statistics taken from small samples. We look at $m$ observations from the Pareto distribution, i.e., $f(x)=p x^{-p-1} I(x \geq 1)$, where $p>0$. Then we observe two order statistics from our sample, i.e., $X_{(i)} \leq X_{(j)}$ for $i<j$. Next we obtain the random variable $R=R_{i j}=X_{(j)} / X_{(i)}$. The density of $R$ is

$$
f_{R}(r)=\frac{p \cdot(m-i) !}{(m-j) !(j-i-1) !}\left(1-r^{-p}\right)^{j-i-1} r^{-p(m-j+1)-1} I(r \geq 1) .
$$

Our goal is to determine whether or not there exist positive constants $a_{n}$ and $b_{N}$ such that $\sum_{n=1}^{N} a_{n} R_{n} / b_{N}$ converges to a nonzero constant in some sense, where $\left\{R_{n}, n \geq 1\right\}$ are i.i.d. copies of $R$. These are called Exact Laws of Large Numbers since they create a fair game situation where the $a_{n} R_{n}$ represent the amount a player wins on the $n^{\text {th }}$ play of some game and $b_{N}-b_{N-1}$ represents the corresponding fair entrance fee for the participant.

\footnotetext{
* E-mail: adler@iit.edu
} 
The results in this paper are quite similar to those that can be found in Adler [2]. Thus all the proofs will be omitted. In that paper the random variable of interest was just the $k^{\text {th }}$ order statistic from a finite sample. If we have i.i.d. Pareto random variables $\left\{X_{n k}, 1 \leq k \leq s, n \geq 1\right\}$, then the $k^{t h}$ order statistic from our $n^{\text {th }}$ sample, $X_{n(k)}$, has the density

$$
f_{X_{n(k)}}(x)=\frac{p \cdot s !}{(s-k) !(k-1) !}\left(1-x^{-p}\right)^{k-1} x^{-p(s-k+1)-1} I(x \geq 1) .
$$

Notice that if we set $m-i=s$ and $m-j=s-k$, which ensures that $j-i=k$, then we can utilize all the results from Adler [2]. As usual we define $\lg x=\log (\max \{e, x\})$ so that we avoid dividing by zero. The first result shows that if $p(m-j+1)<1$ and under an extremely mild condition an Exact Weak Law cannot exist.

Theorem 1. If $p(m-j+1)<1$ and $\max _{1 \leq n \leq N} a_{n}=o\left(b_{N}\right)$, then an Exact Weak Law cannot hold, i.e., the partial sum $\sum_{n=1}^{N} a_{n} R_{n} / b_{N}$ can only converge in probability to zero.

However, if we let $p(m-j+1)=1$, then we can obtain unusual strong laws. But in order to obtain an Exact Strong Law we must select our coefficients and norming sequences properly.

Theorem 2. If $p(m-j+1)=1$, then for all $\beta>0$ we have

$$
\lim _{N \rightarrow \infty} \frac{\sum_{n=1}^{N} \frac{(\lg n)^{\beta-2}}{n} R_{n}}{(\lg N)^{\beta}}=\frac{\left(\begin{array}{c}
m-i \\
j-i-1
\end{array}\right)}{\beta} \text { almost surely. }
$$

When we allow $p(m-j+1)>1$, then typical strong laws exist, since the first moment of our random variable is finite. The mean of that random variable can be found in the following lemma.

Lemma. If $p(m-j+1)>1$, then

$$
E R=\frac{(m-i) ! \Gamma(m-j+1-1 / p)}{(m-j) ! \Gamma(m-i+1-1 / p)} .
$$

In order to obtain these strong laws we can define $\left\{a_{n}, n \geq 1\right\}$ and $\left\{b_{n}, n \geq 1\right\}$ as any pair of positive sequences as long as $b_{n} \uparrow \infty, \sum_{n=1}^{N} a_{n} / b_{N} \rightarrow L$, where $L \neq 0$, and the condition involving $c_{n}=b_{n} / a_{n}$ in each theorem is satisfied. If $L=0$, then these limit theorems still hold, however the limit is zero, which is not terribly interesting.

Theorem 3. If $1<p(m-j+1)<2$ and $\sum_{n=1}^{\infty} c_{n}^{-p(m-j+1)}<\infty$, then

$$
\lim _{N \rightarrow \infty} \frac{\sum_{n=1}^{N} a_{n} R_{n}}{b_{N}}=\frac{L \cdot(m-i) ! \Gamma(m-j+1-1 / p)}{(m-j) ! \Gamma(m-i+1-1 / p)} \quad \text { almost surely. }
$$

Theorem 4. If $p(m-j+1)=2$ and $\sum_{n=1}^{\infty} \lg \left(c_{n}\right) / c_{n}^{2}<\infty$, then

$$
\lim _{N \rightarrow \infty} \frac{\sum_{n=1}^{N} a_{n} R_{n}}{b_{N}}=\frac{L \cdot(m-i) ! \Gamma(m-j+1-1 / p)}{(m-j) ! \Gamma(m-i+1-1 / p)} \quad \text { almost surely. }
$$


Theorem 5. If $p(m-j+1)>2$ and $\sum_{n=1}^{\infty} c_{n}^{-2}<\infty$, then

$$
\lim _{N \rightarrow \infty} \frac{\sum_{n=1}^{N} a_{n} R_{n}}{b_{N}}=\frac{L \cdot(m-i) ! \Gamma(m-j+1-1 / p)}{(m-j) ! \Gamma(m-i+1-1 / p)} \quad \text { almost surely. }
$$

Clearly, in all of these last three theorems the situation of $a_{n}=1$ and $b_{n}=n=c_{n}$ is easily satisfied. Whenever $p(m-j+1)>1$ we have tremendous freedom in selecting our constants. That is certainly not true when $p(m-j+1)=1$.

Next we return to $p(m-j+1)=1$ since there are more unanswered questions. We saw in Theorem 2 that in order to establish an Exact Strong Law we are forced to set $a_{n}$ to be a slowly varying function divided by $\mathrm{n}$, while $b_{n}$ must also be slowly varying. If one wants to try more conventional constants such as $a_{n}=1$ and $b_{n}=n$ we will have to set our sights a bit lower and settle for an Exact Weak Law. The Weak Law can be found in Theorem 6. We then use that Weak Law to obtain the almost sure behavior of our normalized partial sums. It turns out that the weak limit $\left(\begin{array}{c}m-i \\ j-i-1\end{array}\right) /(\alpha+1)$ is the almost sure lower limit of these normalized partial sums. This result is known as a Generalized Law of the Iterated Logarithm.

Theorem 6. If $p(m-j+1)=1$ and $\alpha>-1$, then

$$
\frac{\sum_{n=1}^{N} n^{\alpha} R_{n}}{N^{\alpha+1} \lg N} \stackrel{P}{\rightarrow} \frac{\left(\begin{array}{c}
m-i \\
j-i-1
\end{array}\right)}{\alpha+1} \text {. }
$$

What is interesting about these types of weak laws is that they give a false impression as to what the fair games entrance fee should be. For example in the famous St. Petersburg game, where $P\left\{X_{n}=2^{r}\right\}=2^{-r}, r=1,2, \ldots$, we have from page 252 of Feller [3]

$$
\frac{\sum_{n=1}^{N} X_{n}}{N \lg _{2} N} \stackrel{P}{\rightarrow} 1
$$

where $\lg _{2} N$ is the logarithm to the base two. However, if one would use as the cumulative entrance fee $N \lg _{2} N$, after $N$ plays of that game, then the player would have a decided advantage. We see that via the almost sure behavior of the upper and lower limits of these normalized partial sums

$$
\liminf _{N \rightarrow \infty} \frac{\sum_{n=1}^{N} X_{n}}{N \lg _{2} N}=1 \quad \text { almost surely }
$$

while

$$
\limsup _{N \rightarrow \infty} \frac{\sum_{n=1}^{N} X_{n}}{N \lg _{2} N}=\infty \quad \text { almost surely. }
$$

Thus the player should always play this game. This result can be obtained from Klass and Teicher [4]. Similarly, we have our Generalized Law of the Iterated Logarithm.

Theorem 7. If $p(m-j+1)=1$ and $\alpha>-1$, then

$$
\liminf _{N \rightarrow \infty} \frac{\sum_{n=1}^{N} n^{\alpha} R_{n}}{N^{\alpha+1} \lg N}=\frac{\left(\begin{array}{c}
m-i \\
j-i-1
\end{array}\right)}{\alpha+1} \quad \text { almost surely }
$$


and

$$
\limsup _{N \rightarrow \infty} \frac{\sum_{n=1}^{N} n^{\alpha} R_{n}}{N^{\alpha+1} \lg N}=\infty \quad \text { almost surely. }
$$

The only way to make any of these games fair for both the house and the player is to obtain an Exact Strong Law which can only be achieved by weighting the random variables as we did in Theorem 2, see Adler [1].

\section{Acknowledgment}

I would like to thank the referee for his/her helpful comments which greatly improved upon the presentation of this article and for pointing out an error in the density of $X_{n(k)}$ in Adler [2].

\section{References}

[1] A. Adler: "Exact Strong Laws", Bulletin Institute Mathematics Academia Sinica, Vol. 28(3), (2000), pp. 141-166.

[2] A. Adler: "Exact Laws for Sums of Order Statistics from the Pareto Distrbution", Bulletin Institute Mathematics Academia Sinica, Vol. 31(3), (2003), pp. 181-193.

[3] W. Feller: An Introduction to Probability Theory and Its Applications, Vol. 1, 3rd ed., John Wiley, New York, 1968.

[4] M. Klass and H. Teicher: "Iterated Logarithm Laws for Asymmetric Random Variables Barely With or Without Finite Mean", Annals Probab., Vol. 5(6), (1977), pp. 861-874. 